\title{
Methylation pattern of preferentially expressed antigen of melanoma in acute myeloid leukemia and myelodysplastic syndromes
}

\author{
YA-ZHEN QIN, YAN-HUAN ZHANG, XIAO-YING QIN and HONG-HU ZHU \\ Department of Hematology, Peking University People's Hospital, \\ Peking University Institute of Hematology, Beijing 100044, P.R. China
}

Received September 11, 2015; Accepted January 19, 2017

DOI: $10.3892 / \mathrm{ol} .2017 .5790$

\begin{abstract}
Preferentially expressed antigen of melanoma (PRAME), a tumor-associated antigen, is overexpressed in a variety of hematologic malignancies with a great variation in expression. The majority of patients with acute myeloid leukemia $(A M L)$ 1-eight-twenty one $(E T O)^{+} \mathrm{AML}$ and a certain number of myelodysplastic syndromes (MDS) have an abnormally high increase in $P R A M E$ expression level. The landscape of $P R A M E$ methylation requires evaluation in order to determine the most relevant sites and the exact association of its methylation with expression level and type of disease. In the present study, bone marrow samples collected from 8 AML1-ETO $^{+}$AML, 4 MDS, 3 AML1-ETO- AML and 2 normal volunteers underwent bisulfate sequencing to analyze the methylation status of all four 5'-C-phosphate-G-3' (CpG) regions within the entire PRAME gene. The median PRAME transcript level of 15 patients was $204.5 \%$ (range, 0.02-710.3\%). PRAME transcript levels were inversely associated with the degree of methylation of the -389 to $-146 \mathrm{CpG}$ sites $(\mathrm{r}=-0.69 ; \mathrm{P}=0.002)$ in the $3^{\prime}$ part of the promoter region and the +132 to $+363 \mathrm{CpG}$ sites $(\mathrm{r}=-0.69 ; \mathrm{P}=0.006)$ in the exon $1 \mathrm{~b}$ region. However, not every sample strictly followed this correlation: Certain samples with high degrees of methylation demonstrated abnormally high expression levels, and vice versa. The methylation ratios of $\mathrm{CpG}$ sites in exon 1a were low for all samples (range, $0.0-13.8 \%$ ), and those in exon 2 were similar in 16 samples (range, 72.4-93.4\%), with the exception of one patient with high expression (425.2\%) and significantly low degree of methylation in the PRAME gene (22.2\%). MDS patients revealed similar methylation ratios in the $3^{\prime}$ section
\end{abstract}

Correspondence to: Dr Ya-Zhen Qin, Department of Hematology, Peking University People's Hospital, Peking University Institute of Hematology, 11 Xizhimen South Street, Beijing 100044, P.R. China E-mail: qin2000@aliyun.com

Key words: preferentially expressed antigen of melanoma, methylation, acute myeloid leukemia 1-eight-twenty one, acute myeloid leukemia, myelodysplastic syndromes, bisulfate sequencing of the promoter region, but tended to have lower methylation ratios in the exon $1 \mathrm{~b}$ region $(\mathrm{P}=0.62$ and $\mathrm{P}=0.09$, respectively) compared with those observed in $A M L 1-E T O^{+}$patients with AML and similar degree of PRAME overexpression. Therefore, the hypomethylation of $\mathrm{CpG}$ sites in the 3' part of the promoter region and in exon $1 \mathrm{~b}$ was typically found with PRAME overexpression in AML and MDS. Methylation of other $\mathrm{CpG}$ islands, epigenetic and genetic mechanisms, and type of disease may also be involved.

\section{Introduction}

Preferentially expressed antigen of melanoma (PRAME) was originally identified in a patient with melanoma (1). As a tumor-associated antigen (TAA), PRAME expression level is low in normal tissues and normal bone marrow, but it is highly increased in a variety of solid tumors and hematologic malignancies (1-13). The majority of patients with acute myeloid leukemia (AML) overexpress PRAME (7-11). Furthermore, previous studies have reported that the majority of patients with myelodysplastic syndromes (MDS) overexpress PRAME (12,13). A high level of variation exists in the PRAME transcript levels for patients with AML and MDS (8-13). In particular, the majority of patients with $A M L 1$-eight-twenty one $(E T O)^{+} \mathrm{AML}$ and a number of patients with MDS overexpress PRAME with abnormally high increases $(10,12,13)$. PRAME transcript levels detected by reverse transcription-quantitative polymerase chain reaction (RT-qPCR) or quantitative PCR (qPCR) have been clinically used to determine the prognosis and monitor minimal residual disease (8-13). In addition to its clinical usefulness, the molecular mechanism of PRAME overexpression has also been investigated.

Epigenetic events appear to be the main mechanism underlying the regulation of gene expression $(14,15)$. It has previously been revealed that DNA methylation is the main restricting factor for TAA expression (16). Ikeda et al (1) first identified the antigen PRAME and revealed that the demethylating agent 5-aza-2'-deoxycytidine could activate $P R A M E$ gene expression, which implied that DNA methylation was involved in the regulation of its expression. Subsequent studies confirmed this result in AML and chronic myeloid leukemia (CML) (17-22). However, despite numerous studies 
demonstrating an inverse correlation between methylation of specific 5'-C-phosphate-G-3' (CpG) sites and PRAME expression, the $\mathrm{CpG}$ island regions studied were different (17-20). Furthermore, to the best of our knowledge, no studies have analyzed all the $\mathrm{CpG}$ sites of the PRAME gene. The landscape of PRAME gene methylation remains to be elucidated.

Cloning sequencing of sodium bisulfate-treated DNA is a classical method used to directly elucidate the methylation status of every $\mathrm{CpG}$ site. As a result of its complexity, the sample number is typically small (17-20). Therefore, although a general correlation between the hypomethylation of specific CpG sites of PRAME and its overexpression was revealed (17-20), whether the degree of hypomethylation is associated with the degree of increase in its expression remains unknown. Furthermore, the association between hypomethylation of $\mathrm{CpG}$ sites and PRAME overexpression has not been specifically investigated in $A M L 1-E T O^{+}$AML and MDS.

The present study performed bisulfate sequencing of four $\mathrm{CpG}$ island regions in bone marrow samples collected from 15 patients with AML and MDS and 2 normal volunteers. The present study demonstrated that PRAME expression level was associated with the methylation status of specific $\mathrm{CpG}$ sites and that their degree of methylation was generally, but not precisely, associated with PRAME transcript levels.

\section{Materials and methods}

Samples. In total, 17 bone marrow samples, which were collected between January 2010 and November 2011 at the Peking University Institute of Hematology (Beijing, China), were included in the present study. The bone marrow of 15 patients was aspirated prior to treatment. The patients' median age was 47 years (range, 25-80 years), and 8 were male while 7 were female. A total of 3 patients were AML1-ETO- AML, 8 were AML1-ETO ${ }^{+}$AML, 3 were MDS-refractory anemia with excess blasts 2 (RAEB2) and 1 was MDS-refractory cytopenia with multilineage dysplasia (RCMD). The diagnosis was based on morphological, immunophenotyping, cytogenetic and molecular studies according to the 2008 World Health Organization classification (23). A total of 2 bone marrow samples were obtained from normal volunteers. The present study was approved by the ethics committee of Peking University Peoples' Hospital (Beijing, China). Written informed consent was obtained from all patients prior to enrollment in the present study.

RNA extraction and complementary DNA (cDNA) synthesis. Mononuclear cells were isolated from bone marrow samples by Ficoll-Hypaque gradient centrifugation (TBD Science, Tianjin, China) at $500 \mathrm{x} g$ for $20 \mathrm{~min}$ at room temperature. TRIzol ${ }^{\circledR}$ Reagent (Invitrogen; Thermo Fisher Scientific, Inc., Waltham, MA, USA) was used to extract total RNA according to the manufacturer's instructions. A High-Capacity cDNA Reverse Transcription kit (Applied Biosystems; Thermo Fisher Scientific, Inc.) was used to synthesize cDNA from RNA.

Detection of PRAME transcript levels. TaqMan-based qPCR was performed as previously described $(5,10,12)$. Abelson tyrosine-protein kinase 1 (ABL) was selected as a control gene (24). Primers and probe for PRAME were reported previously $(10,12)$, and those for ABL were previously reported by Beillard et al (24). The experiments were performed in duplicates. The transcript level was calculated as: transcript level=PRAME transcript copies/ABL copies $\times 100 \%$.

Bisulfate treatment of genomic DNA. DNAzol ${ }^{\circledR}$ reagent (Invitrogen; Thermo Fisher Scientific, Inc.) was used to extract genomic DNA from mononuclear cells. In order to perform methylation studies, genomic DNA was treated with sodium bisulfate to convert all methyl-free cytosines into uracils, whereas methylated cytosines remained unchanged. For each sample, 1-3 $\mu \mathrm{g}$ genomic DNA was dissolved in $18 \mu \mathrm{l}$ sterile water, $2 \mu 13 \mathrm{~N} \mathrm{NaOH}$ was added and the samples were denatured for $20 \mathrm{~min}$ at $42^{\circ} \mathrm{C}$. In order to convert unmethylated cytosines, denatured DNA was incubated in $50 \mu 10.1 \mathrm{M}$ hydroquinone (Sigma-Aldrich; EMD Millipore, Billerica, MA, USA) and $350 \mu 13 \mathrm{M}$ sodium bisulfate (Sigma-Aldrich; EMD Millipore) for $16 \mathrm{~h}$ at $50^{\circ} \mathrm{C}$. The Wizard ${ }^{\circledR}$ DNA Clean-Up System (Promega Corporation, Madison, WI, USA) was used to purify modified DNA, and the DNA was eluted in $40 \mu \mathrm{l}$ sterile water. Subsequently, $5 \mu 13 \mathrm{~N} \mathrm{NaOH}$ was added and incubated for $15 \mathrm{~min}$ at $37^{\circ} \mathrm{C}$ to complete the DNA modification. A total of $75 \mu 15 \mathrm{M}$ ammonium acetate and $300 \mu \mathrm{l}$ pure ethanol was then added, followed by centrifugation at 9,600 $\mathrm{x} g$ for $20 \mathrm{~min}$ at $4^{\circ} \mathrm{C}$. The DNA was washed with $200 \mu 170 \%$ ethanol. The DNA pellet was dissolved in $20 \mu 1$ sterile water.

CpG island identification and polymerase chain reaction (PCR) amplification for methylation analysis. A CpG island was defined by Gardiner-Garden and Frommer (25) as the observed $\mathrm{CpG} /$ expected $\mathrm{CpG}$ ratio $\geq 0.6$, with a $\mathrm{GC}$ content $>50 \%$ of a 200 bp sequence. $\mathrm{CpG}$ islands of the PRAME gene (GeneBank: NG 000002.1; https://www.ncbi.nlm.nih. gov/nucleotide/18860922) were identified by submitting its sequence to www.bio-soft.net/sms/cpg_island.html. A total of four $\mathrm{CpG}$ islands, which were individually located in the promoter, exon 1a, exon $1 \mathrm{~b}$ and exon 2 regions, respectively, were identified.

AmpliTaq Gold ${ }^{\circledR} 360$ DNA Polymerase (Applied Biosystems; Thermo Fisher Scientific, Inc.) was used for PCR amplification. The primer pairs for the amplification of the exon $1 \mathrm{~b}$ region reported by Ortmann et al (19) were used, and those for the promoter, exon 1a and exon 2 regions were designed in house and are presented in Table I. The PCR conditions were as follows: Incubation at $95^{\circ} \mathrm{C}$ for $10 \mathrm{~min}$, followed by 11 cycles of touch-down cycling at $95^{\circ} \mathrm{C}$ for $30 \mathrm{sec}$, annealing from $63-56^{\circ} \mathrm{C}$ with a $0.7^{\circ} \mathrm{C}$ decrease/cycle for $30 \mathrm{sec}$ and incubation at $72^{\circ} \mathrm{C}$ for $60 \mathrm{sec}, 30$ cycles at $95^{\circ} \mathrm{C}$ for $30 \mathrm{sec}$ and incubation at $56^{\circ} \mathrm{C}$ for $30 \mathrm{sec}$ and $72^{\circ} \mathrm{C}$ for $60 \mathrm{sec}$, and a final 10 -min incubation at $72^{\circ} \mathrm{C}$. Each PCR reaction was performed in duplicates. Amplification products were separated by electrophoresis on a $1.2 \%$ agarose gel and purified. Subsequently, the purified duplicates were pooled together and cloned into a pCR2.1 plasmid vector (Invitrogen; Thermo Fisher Scientific, Inc.). Next, DH5a competent cells (Invitrogen; Thermo Fisher Scientific, Inc.) were transformed according to the manufacturer's instructions, and 10 clones/PCR products were sequenced using primers (forward, 5'-TGTAAAACGACGGCCAGT-3' and reverse primer, 5'-CAGGAAACAGCTATGACC-3'). The 
Table I. Polymerase chain reaction primer sequences and product sizes.

Amplification region

Primer sequences

Product

Amplification region

Promoter

5'-GTTGAATGTTTTAAGTAGAGAGGG-3' (F)

size (bp)

Exon 1a

5'-TATATACAAAACCCACTTCCTC-3' (R)

Exon la 5'-AGAGGGTTTGGGAGGAAGTG-3' (F)

5'-CTCCACCCTACTTTCCCTACA-3' (R)

Exon 2

5'-TTTTGGG(C/T)GATTTTATTGGA-3' (F)

5'-CCССТАТАATCAAAAACCCTAACT-3' (R)

610

F, forward; R, reverse.

methylation ratio, expressed as a percentage, is defined as the ratio of methylated $\mathrm{CpG}$ sites/all included $\mathrm{CpG}$ sites.

Statistical analysis. Statistical analysis was performed using SPSS software version 11.5 (SPSS, Inc., Chicago, IL, USA) and GraphPad Prism 5 (Graphpad Software, Inc., La Jolla, CA, USA). The association between two variables was indicated by Spearman correlation coefficient $r$ and analyzed by Spearman's non-parametric correlations. The Mann-Whitney non-parametric $U$ test was used to compare the levels of the PRAME transcript between two groups. P-values were from two-tailed tests and $\mathrm{P}<0.05$ was considered to indicate a statistically significant difference.

\section{Results}

PRAME transcript levels. Figs. 1 and 2 present the PRAME transcript levels of 17 samples. Two normal bone marrow samples exhibited PRAME transcript levels of 0.1 and $0.2 \%$, respectively. The median $P R A M E$ transcript level of the 15 patients was $204.5 \%$ (range, $0.02-710.3 \%$ ) with the highest level 35,515 times compared with the lowest level.

Methylation patterns. As presented in Figs. 1 and 2, the methylation patterns of the four $\mathrm{CpG}$ island regions varied among the patients. For the -389 to $-146 \mathrm{CpG}$ sites in the 3 'end of the promoter region and the +132 to $+363 \mathrm{CpG}$ sites in the exon $1 \mathrm{~b}$ region, the degree of methylation decreased as PRAME transcript levels increased. However, the degree of methylation was observed to be similar among all samples in the 5' end of the promoter (Fig. 1, -540 to -420 sites), exon 1a (Fig. 1, +3 to +105 sites) and exon 2 regions (Fig. $2,+1542$ to +2118 sites). Almost all $\mathrm{CpG}$ sites in the exon 1a region were unmethylated for every sample despite whether the PRAME transcript levels were high or low.

Subsequently, methylation ratios were determined. As presented in Table II, the methylation ratios of all CpG sites were significantly inversely associated with the PRAME transcript levels $(\mathrm{P}=0.002)$. Furthermore, the methylation ratios of $\mathrm{CpG}$ sites in the promoter and exon $1 \mathrm{~b}$ regions, but not those of the exon 1a or exon 2 regions, were significantly negatively associated with PRAME transcript levels (all $\mathrm{P}<0.05)$. Therefore, $P R A M E$ transcript levels were negatively
Table II. Correlations between PRAME transcript levels and methylation ratios of the four $\mathrm{CpG}$ island regions.

\begin{tabular}{lccc}
\hline Region & CpG sites & r & P-value \\
\hline Promoter & -540 to -130 & -0.52 & $0.03^{\mathrm{a}}$ \\
3' Part of promoter & -389 to -146 & -0.69 & $0.00^{\mathrm{a}}$ \\
Exon 1a & +4 to +105 & -0.29 & 0.26 \\
Exon 1b & +132 to +363 & -0.63 & $0.01^{\mathrm{a}}$ \\
Exon 2 & $+1,542$ to $+2,118$ & -0.38 & 0.13 \\
Total & -540 to $+2,118$ & -0.86 & $0.00^{\mathrm{a}}$ \\
\hline
\end{tabular}

${ }^{a} \mathrm{P}<0.05$. PRAME, preferentially expressed antigen of melanoma; CpG, 5'-C-phosphate-G-3'.

associated with the degree of methylation. The methylation ratios in the exon la region were low for all samples (median, $1.3 \%$; range, $0.0-13.8 \%$ ), and those in the exon 2 region were similar in 16 patients (median, 88.9\%; range, 72.4-93.4\%), with the exception of 1 patient (No. P13), who exhibited a significantly low degree of methylation (22.2\%). The association between PRAME transcript levels and the degree of methylation of individual $\mathrm{CpG}$ sites in the promoter and exon $1 \mathrm{~b}$ regions were further analyzed. As presented in Table III, for the majority of the $\mathrm{CpG}$ sites ( -389 to -146 and +132 to $+363)$, there were significant negative correlations between the degree of methylation of individual $\mathrm{CpG}$ sites and PRAME transcript levels, but these correlations were not evident for the -540 to $-420 \mathrm{CpG}$ sites. Therefore, PRAME transcript levels were inversely associated with the degree of methylation of -389 to $-146 \mathrm{CpG}$ sites in the promoter region and +132 to +363 $\mathrm{CpG}$ sites in the exon $1 \mathrm{~b}$ region.

As presented in Fig. 3A, all 17 bone marrow samples were arranged according to $P R A M E$ transcript levels from low to high. This intuitively revealed that, although $P R A M E$ transcript levels were negatively associated with the degree of methylation, not all samples obeyed this rule: Patients P3 and P6 demonstrated relatively low degrees of methylation, and patients P10 and P14 displayed relatively high degrees of methylation, compared with the general tendency of all the patients (Figs. 1,2 and 3A). This was also the case for the individual $\mathrm{CpG}$ sites in both the 3 ' part 


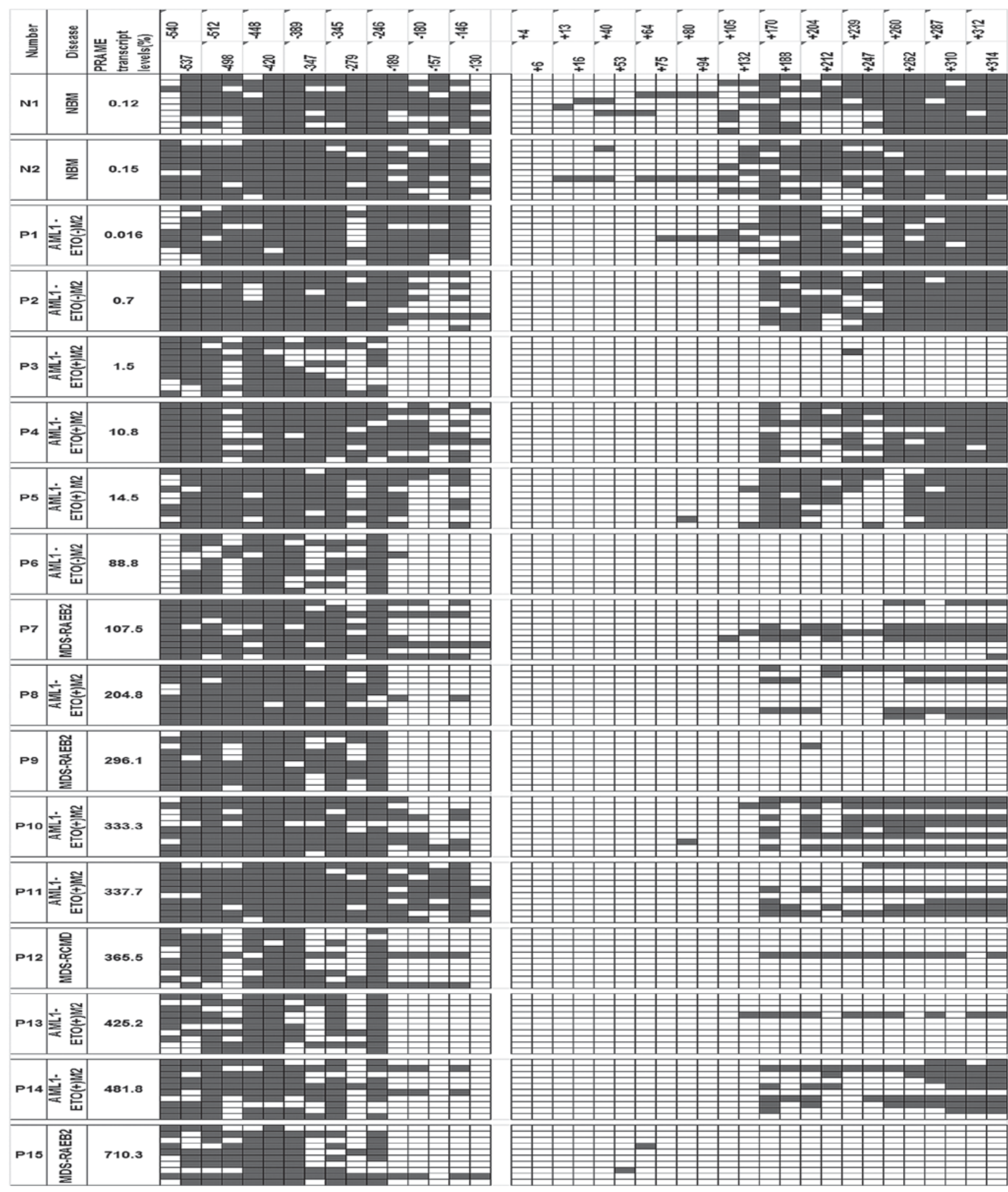

Figure 1. Clone sequencing results of -540 to $+314 \mathrm{CpG}$ sites of 17 bone marrow samples from 2 normal volunteers and 15 patients. White color represents unmethylated cytosines; black color represents methylated cytosines. CpG, 5'-C-phosphate-G-3'; PRAME, preferentially expressed antigen of melanoma; NBM, normal bone marrow; AML, acute myeloid leukemia; MDS, myelodysplastic syndromes; ETO, eighty twenty one; P, patient; N, normal; RAEB2, MDS-refractory anemia with excess blasts 2; RCMD, MDS-refractory cytopenia with multilineage dysplasia.

of the promoter and the exon $1 \mathrm{~b}$ regions. Therefore, a one-to-one correlation did not exist between the degree of PRAME methylation and its transcript level.

Comparisons of the degree of methylation of $C p G$ sites between different regions and between patients with AML and
$M D S$. As presented in Fig. 3A, the methylation ratio of the +132 to $+363 \mathrm{CpG}$ sites was closely associated with that of the -389 to -346 sites for all the samples $(\mathrm{r}=0.88 ; \mathrm{P}<0.0001)$. This demonstrated that the degree of methylation of the sites in the 3 'part of the promoter was concordant with that of the sites in exon $1 \mathrm{~b}$ regions of the PRAME gene. 


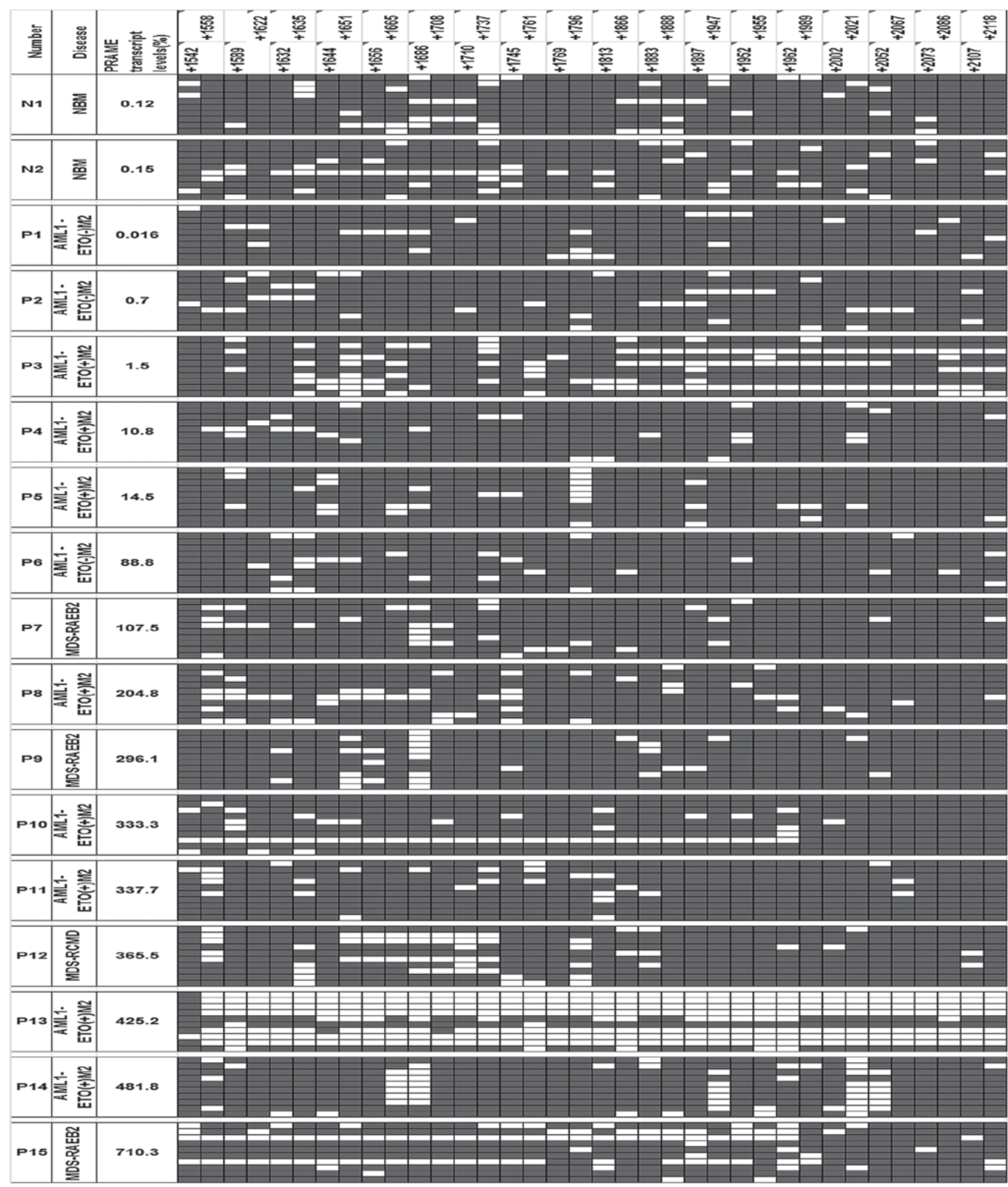

Figure 2. Clone sequencing results of $+1,542$ to $+2,118 \mathrm{CpG}$ sites of 17 bone marrow samples from 2 normal volunteers and 15 patients. White color represents unmethylated cytosines; black color represents methylated cytosines. CpG, 5'-C-phosphate-G-3'; PRAME, preferentially expressed antigen of melanoma; NBM, normal bone marrow; AML, acute myeloid leukemia; MDS, myelodysplastic syndromes; ETO, eighty twenty one; P, patient; N, normal; RAEB2, MDS-refractory anemia with excess blasts 2; RCMD, MDS-refractory cytopenia with multilineage dysplasia.

Fig. 3B presents the association between PRAME transcript levels and the methylation ratios of the -389 to -146 and +132 to $+363 \mathrm{CpG}$ sites in patients with AML1-ETO ${ }^{+} \mathrm{AML}$ and MDS. The degree of methylation of the -389 to $-146 \mathrm{CpG}$ sites was similar in comparison with that of the +132 to +363 sites in patients with $A M L 1-E T O^{+}$AML $(\mathrm{P}=0.46)$. On the contrary, the degree of methylation of the +132 to $+363 \mathrm{CpG}$ sites was significantly lower compared with that of the -389 to -146 sites in patients with MDS $(\mathrm{P}=0.021)$.

The present study also compared AML1-ETO AML and MDS. A total of 9 patients (P7-P15) were included to ensure similar PRAME transcript levels between the patients (median, 
Table III. Correlations between PRAME transcript levels and methylation ratios of the individual $\mathrm{CpG}$ sites in 17 samples.

\begin{tabular}{ccccccc}
\hline \multicolumn{2}{c}{-540 to $-130 \mathrm{CpG}$ sites } & & \multicolumn{3}{c}{+132 to $+363 \mathrm{CpG}$ sites } \\
\cline { 1 - 1 } \cline { 6 - 7 } Site & $\mathrm{r}$ & P-value & & Site & $\mathrm{r}$ & P-value \\
\hline-540 & 0.24 & 0.35 & & +132 & -0.42 & 0.09 \\
-537 & -0.27 & 0.30 & & +170 & -0.52 & $0.03^{\mathrm{a}}$ \\
-512 & 0.19 & 0.47 & & +188 & -0.57 & $0.02^{\mathrm{a}}$ \\
-498 & -0.25 & 0.33 & & +204 & -0.55 & $0.02^{\mathrm{a}}$ \\
-448 & 0.32 & 0.20 & & +212 & -0.66 & $0.00^{\mathrm{a}}$ \\
-420 & 0.00 & 1.00 & & +239 & -0.58 & $0.01^{\mathrm{a}}$ \\
-389 & -0.59 & $0.01^{\mathrm{a}}$ & & +247 & -0.45 & 0.07 \\
-347 & -0.62 & $0.01^{\mathrm{a}}$ & +260 & -0.54 & $0.03^{\mathrm{a}}$ \\
-345 & -0.31 & 0.22 & & +262 & -0.66 & $0.00^{\mathrm{a}}$ \\
-279 & -0.60 & $0.01^{\mathrm{a}}$ & +287 & -0.56 & $0.02^{\mathrm{a}}$ \\
-246 & -0.47 & 0.07 & +310 & -0.55 & $0.02^{\mathrm{a}}$ \\
-189 & -0.63 & $0.01^{\mathrm{a}}$ & +312 & -0.57 & $0.02^{\mathrm{a}}$ \\
-180 & -0.54 & $0.02^{\mathrm{a}}$ & +314 & -0.56 & $0.02^{\mathrm{a}}$ \\
-157 & -0.67 & $0.00^{\mathrm{a}}$ & +342 & -0.58 & $0.02^{\mathrm{a}}$ \\
-146 & -0.63 & $0.01^{\mathrm{a}}$ & +363 & -0.57 & $0.02^{\mathrm{a}}$ \\
-130 & -0.38 & 0.13 & & & \\
\hline
\end{tabular}

${ }^{\mathrm{a}} \mathrm{P}<0.05$. PRAME, preferentially expressed antigen of melanoma; CpG, 5'-C-phosphate-G-3' .

AML1-ETO ${ }^{+}$AML vs. MDS, 337.7 vs. $\left.330.8 \% ; \mathrm{P}=0.81\right)$. Patients with $A M L 1-E T O^{+}$AML demonstrated higher methylation ratios of the +132 to +363 sites but similar methylation ratios of the -389 to -146 sites, compared with those in patients with MDS ( $\mathrm{P}=0.09$ and $\mathrm{P}=0.62$, respectively). Therefore, the role of the methylation status of $\mathrm{CpG}$ sites in the promoter and exon $\mathrm{bb}$ regions may vary between $A M L 1-E T O^{+}$AML and MDS.

\section{Discussion}

The physiological role of $P R A M E$ remains to be elucidated. Previous studies have suggested that PRAME serves a role in the tumorigenic process due to its involvement in growth and survival, although the results are conflicting (26-28). Overexpression of PRAME is frequently observed in various human cancer types, including leukemic malignancies (1-13). Therefore, the mechanism of its overexpression requires further clarification in order to improve therapeutic treatment. Methylation at DNA CpG islands is an important mechanism that regulates gene expression (14). In the present study, using the bisulfate sequencing method, it was demonstrated that PRAME expression was associated with the methylation status of specific $\mathrm{CpG}$ sites, and that the degree of hypomethylation was generally inversely associated with its transcript levels in AML and MDS.

Numerous studies have demonstrated that the hypomethylation of PRAME is correlated with its overexpression (17-20); however, the results are inconsistent, and certain issues still require clarification. Bisulfate sequencing is a direct and comprehensive but complex method used for methylation studies. Therefore, previous studies that analyzed PRAME methylation in leukemia performed clone sequencing in 5 cell lines, 2 normal controls and 22 patient samples, but did not include the entire $\mathrm{CpG}$ island regions (17-20). Along with the individual variances between patients, the exact assessment of the methylation effect may be difficult to determine and may produce biased results. In the present study, bone marrow samples collected from 15 newly diagnosed patients and 2 normal volunteers with coverage of 4.6-log of PRAME transcript levels were included, in order to make the results more definite. To the best of our knowledge, the present study included the largest sample number and $\mathrm{CpG}$ island regions in order to investigate PRAME methylation by bisulfate sequencing.

The region and $\mathrm{CpG}$ site that are most associated with $P R A M E$ expression remain controversial. Roman-Gomez et al (18) analyzed the methylation status in exon 2 in the chronic phase of blast crisis CML. Schenk et al (17) investigated both the promoter and exons $1 \mathrm{a}$ and $1 \mathrm{~b}$ regions in AML, and demonstrated that PRAME transcription required the demethylation of two regions: One in the promoter (between -189 and -130) and one in exon $1 b$. Ortmann et al (19) analyzed the methylation status of exon $1 \mathrm{~b}$ in AML and revealed that its hypomethylation induced aberrant PRAME expression. Gutierrez-Cosío et al (20) observed an inverse correlation between PRAME messenger RNA levels and methylation status in AML by analyzing the promoter region. The present study searched the entire PRAME gene and identified four $\mathrm{CpG}$ island regions, all of which were individually reported by others. However, the present study performed bisulfate sequencing for all these islands to comprehensively evaluate the PRAME methylation status. Statistical analysis indicated that PRAME methylation is generally relevant to its transcript levels. Furthermore, the degree of methylation of $\mathrm{CpG}$ sites in the 3'end of the promoter and the whole exon $1 \mathrm{~b}$ regions are significantly negatively associated with PRAME transcript levels. The present study also demonstrated that a close association exists between these two methylation regions in individual patients. Although the relevant regions investigated were in accordance with a study by Schenk et al (17), another previous study did not analyze exon 2, which was identified as being relevant, according to Roman-Gomez et al (18).

In addition, a relatively higher number samples were analyzed in the present study compared with previous reports (17-20), and thus certain exceptions were revealed. Firstly, the degree of methylation of the relevant two regions was not always inversely correlated to PRAME expression. In the present study, in certain samples methylation degree and expression level were not negatively correlated. A number of samples with high levels of PRAME methylation exhibited high expression and vice versa. Secondly, the methylation characteristics of exon 2 for the majority of patients was identified as a low proportion of methylation and no specific sites of $\mathrm{CpG}$ methylation; however, a patient with $A M L 1-E T O^{+} \mathrm{AML}$ and abnormally high $P R A M E$ expression level revealed a unique pattern; almost all $\mathrm{CpG}$ sites were unmethylated in $7 / 10$ clones. These exceptions implied that the degree of methylation in the 3 'end of the promoter and exon $1 \mathrm{~b}$ regions may serve an important role, but may not be the only mechanism determining PRAME transcript levels. The observation that the gene 

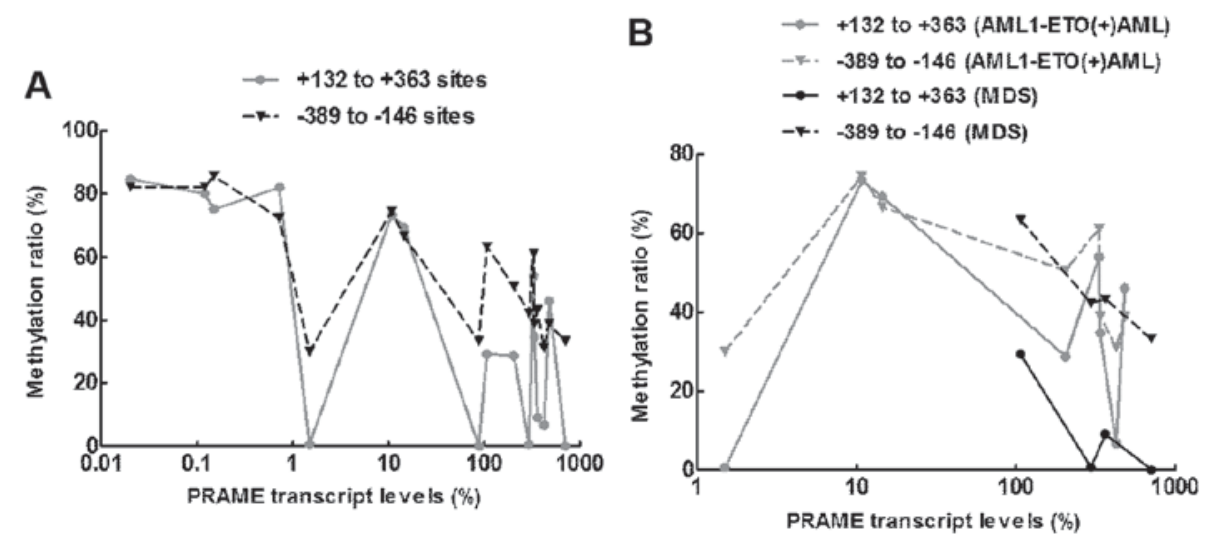

Figure 3. Correlations between PRAME transcript levels and methylation ratios. (A) Correlations between PRAME transcript levels and methylation ratios of -389 to -146 and +132 to $+363 \mathrm{CpG}$ sites of all samples. (B) Comparisons of PRAME methylation ratios of -389 to -146 and +132 to +363 CpG sites between AML1-ETO ${ }^{+}$AML and MDS. CpG, 5'-C-phosphate-G-3'; PRAME, preferentially expressed antigen of melanoma; AML, acute myeloid leukemia; MDS, myelodysplastic syndromes; ETO, eighty twenty one.

expression level was not associated with its degree of methylation was also reported for other genes (29). Methylation in other $\mathrm{CpG}$ islands and mechanisms, including histone modifications, small RNA and genetic abnormalities, may be involved $(30,31)$; this involvement requires further investigation.

It has previously been revealed the majority of patients with AML1-ETO+ AML and a certain number of patients with MDS overexpressed PRAME with abnormally high increases $(10,12,13)$; however, to the best of our knowledge, no previous studies have investigated the effect of methylation on PRAME overexpression. Previous studies reported that the methylation pattern may be associated with the subtype of disease (32). In the present study, the comparison performed between patients with AML1-ETO ${ }^{+}$AML and MDS with comparably high PRAME transcript levels demonstrated that their methylation patterns were not the same. The degrees of methylation of the two relevant regions were similar for AML1-ETO ${ }^{+}$AML, but different for patients with MDS. As a result, there was a tendency that the degree of methylation in AML1-ETO ${ }^{+}$AML was higher compared with that in patients with MDS. This implied that the effect of methylation on the expression of PRAME may vary between these two types of malignancies, and PRAME overexpression was more associated with hypomethylation of exon $1 \mathrm{~b}$ compared with the promoter region in patients with MDS. A greater number of samples should be analyzed by methods such as quantitative methylation-specific PCR, in order to confirm this result. The degree of regulation of gene expression by methylation may be associated with the type of disease. Different types of leukemia may also be the reason for the variety of methylation regions, as demonstrated in previous studies (17-20).

The cellular composition of bone marrow is heterogeneous, and contains blast cells, cells in different stages of differentiation and mature cells, including myelocytes, metamyelocytes, neutrophils, monocytes, erythrocytes and lymphocytes (23). The present study did not sort blast cells to perform bisulfate sequencing. This is mainly because $\mathrm{CD} 34^{+}$cells could not represent the whole abnormal cell population in AML1-ETO+ M2 and MDS. It was previously reported that blast cells [cluster of differentiation (CD) $34^{+}$] and immature myeloid cells, nucleated erythrocytes and lymphocytes (all CD34-), abnormally overexpressed PRAME and Wilms tumor 1 in MDS (12). In addition, certain $A M L 1-E T O^{+} \mathrm{M} 2$ cases are characterized by an abundance of abnormal myelocytes, which are CD34. Furthermore, the present study demonstrated that the methylation degree among clones for sequencing is consistent in the majority of samples. This implied that normal cells had little impact on the evaluation. Therefore, mononuclear cells may be better than blast cells for the evaluation of methylation status in $A M L 1-E T O^{+} \mathrm{M} 2$ and MDS.

In conclusion, the methylation status of $\mathrm{CpG}$ sites in the 3'part of the promoter and the exon $1 \mathrm{~b}$ region was generally associated with PRAME expression level in AML and MDS. Furthermore, its effects were important, but did not comprise the sole mechanism regulating PRAME transcript levels; this may also be associated with the type of disease. Extensive investigations into the effect of methylation of other $\mathrm{CpG}$ islands, as well as other epigenetic and genetic mechanisms, on the regulation of PRAME expression are required. The current study facilitates the comprehensive understanding of the mechanisms underlying the regulation of PRAME expression, which may help to improve targeted therapy for hematologic malignancies.

\section{Acknowledgements}

The present study was supported by the Nature Science Foundation of China (grant nos. 81170483, 81570130 and 81370639).

\section{References}

1. Ikeda H, Lethé B, Lehmann F, van Baren N, Baurain JF, de Smet C, Chambost H, Vitale M, Moretta A, Boon T and Coulie PG: Characterization of an antigen that is recognized on a melanoma showing partial HLA loss by CTL expressing an NK inhibitory receptor. Immunity 6: 199-208, 1997.

2. Neumann E, Engelsberg A, Decker J, Störkel S, Jaeger E, Huber C and Seliger B: Heterogenous expression of the tumor-associated antigens RAGE-1, PRAME, and glycoprotein 75 in human renal cell carcinoma: Candidates for T-cell-based immunotherapies? Cancer Res 58: 4090-4095, 1998.

3. Proto-Siqueira R, Falcão RP, de Souza CA, Ismael SJ and Zago MA: The expression of PRAME in chronic lymphoproliferative disorders. Leuk Res 27: 393-396, 2003. 
4. Radich JP, Dai H, Mao M, Oehler V, Schelter J, Druker B, Sawyers C, Shah N, Stock W, Willman CL, et al: Gene expression changes associated with progression and response in chronic myeloid leukaemia. Proc Natl Acad Sci USA 103: 2794-2799, 2006.

5. Qin Y, Lu J, Bao L, Zhu H, Li J, Li L, Lai Y, Shi H, Wang Y, Liu Y, et al: Bortezomib improves progression-free survival in multiple myeloma patients overexpressing preferentially expressed antigen of melanoma. Chin Med J (Engl) 127: $1666-1671,2014$

6. Abdelmalak CA, Yahya RS, Elghannam DM, El-Khadragy AE and Abd El Messih HM: PRAME gene expression in childhood acute lymphoblastic leukemia: Impact on prognosis. Clin Lab 60 55-61, 2014.

7. van Baren N, Chambost H, Ferrant A, Michaux L, Ikeda H, Millard I, Olive D, Boon T and Coulie PG: PRAME, a gene encoding an antigen recognized on a human melanoma by cytolytic T cells, is expressed in acute leukemia cells. Br J Haematol 102: 1376-1379, 1998.

8. Santamaría C, Chillón MC, García-Sanz R, Balanzategui A, Sarasquete ME, Alcoceba M, Ramos F, Bernal T, Queizán JA, Peñarrubia MJ, et al: The relevance of preferentially expressed antigen of melanoma (PRAME) as a marker of disease activity and prognosis in acute promyelocytic leukemia. Haematologica 93: $1797-1805,2008$

9. Santamaría CM, Chillón MC, García-Sanz R, Pérez C, Caballero MD, Ramos F, de Coca AG, Alonso JM, Giraldo P, Bernal T, et al: Molecular stratification model for prognosis in cytogenetically normal acute myeloid leukemia. Blood 114 $148-152,2009$

10. Qin Y, Zhu H, Jiang B, Li J, Lu X, Li L, Ruan G, Liu Y, Chen S and Huang X: Expression patterns of WT1 and PRAME in acute myeloid leukemia patients and their usefulness for monitoring minimal residual disease. Leuk Res 33: 384-390, 2009.

11. Steinbach D, Bader P, Willasch A, Bartholomae S, Debatin KM, Zimmermann M, Creutzig U, Reinhardt D and Gruhn B: Prospective validation of a new method of monitoring minimal residual disease in childhood acute myelogenous leukemia. Clin Cancer Res 21: 1353-1359, 2015.

12. Qin YZ, Zhu HH, Liu YR, Wang YZ, Shi HX, Lai YY, Xu LP, Liu DH, Jiang Q, Li LD, et al: PRAME and WT1 transcripts constitute a good molecular marker combination for monitoring minimal residual disease in myelodysplastic syndromes. Leuk Lymphoma 54: 1442-1449, 2013.

13. Liberante FG, Pellagatti A, Boncheva V, Bowen DT, Mills KI, Boultwood J and Guinn BA: High and low, but not intermediate, PRAME expression levels are poor prognostic markers in myelodysplastic syndrome at disease presentation. Br J Haematol 162: 282-285, 2013.

14. Bird A: DNA methylation patterns and epigenetic memory. Genes Dev 16: 6-21, 2002.

15. You JS and Jones PA: Cancer genetics and epigenetics: Two sides of the same coin? Cancer Cell 22: 9-20, 2012.

16. Meklat F, Li Z, Wang Z, Zhang Y, Zhang J, Jewell A and Lim SH: Cancer-testis antigens in haematological malignancies. Br J Haematol 136: 769-776, 2007.

17. Schenk T, Stengel S, Goellner S, Steinbach D and Saluz HP: Hypomethylation of PRAME is responsible for its aberrant overexpression in human malignancies. Genes Chromosomes Cancer 46: 796-804, 2007.

18. Roman-Gomez J, Jimenez-Velasco A, Agirre X, Castillejo JA, Navarro G, Jose-Eneriz ES, Garate L, Cordeu L, Cervantes F, Prosper F, et al: Epigenetic regulation of PRAME gene in chronic myeloid leukemia. Leuk Res 31: 1521-1528, 2007.
19. Ortmann CA, Eisele L, Nückel H, Klein-Hitpass L, Führer A, Dührsen U and Zeschnigk M: Aberrant hypomethylation of the cancer-testis antigen PRAME correlates with PRAME expression in acute myeloid leukemia. Ann Hematol 87: 809-818, 2008.

20. Gutierrez-Cosío S, de la Rica L, Ballestar E, Santamaría C, Sánchez-Abarca LI, Caballero-Velazquez T, Blanco B, Calderón C, Herrero-Sánchez C, Carrancio S, et al: Epigenetic regulation of PRAME in acute myeloid leukemia is different compared to CD34+ cells from healthy donors: Effect of 5-AZA treatment. Leuk Res 36: 895-899, 2012.

21. Atanackovic D, Luetkens T, Kloth B, Fuchs G, Cao Y, Hildebrandt Y, Meyer S, Bartels K, Reinhard H, Lajmi N, et al: Cancer-testis antigen expression and its epigenetic modulation in acute myeloid leukemia. Am J Hematol 86: 918-922, 2011.

22. Luetkens T, Schafhausen P, Uhlich F, Stasche T, Akbulak R, Bartels BM, Hildebrandt Y, Gontarewicz A, Kobold S, Meyer S, et al: Expression, epigenetic regulation, and humoral immunogenicity of cancer-testis antigens in chronic myeloid leukemia. Leuk Res 34: 1647-1655, 2010.

23. Swerdlow SH, Campo E, Harris NL, Jaffe ES, Pileri SA and Stein H (eds): WHO Classification of Tumours of Haematopoietic and Lymphoid Tissues. 4th Edition. International Agency for Research on Cancer (IARC), Lyon, pp87-110, 2008.

24. Beillard E, Pallisgaard N, van der Velden VH, Bi W, Dee R, van der Schoot E, Delabesse E, Macintyre E, Gottardi E, Saglio G, et al: Evaluation of candidate control genes for diagnosis and residual disease detection in leukemic patients using 'real-time' quantitative reverse-transcriptase polymerase chain reaction (RQ-PCR)-a Europe against cancer program. Leukemia 17: 2474-2486, 2003.

25. Gardiner-Garden $\mathrm{M}$ and Frommer $\mathrm{M}: \mathrm{CpG}$ islands in vertebrate genomes. J Mol Biol 196: 261-282, 1987.

26. Epping MT, Wang L, Edel MJ, Carlee L, Hernandez M and Bernards R: The human tumor antigen PRAME is a dominantrepressor of retinoic acid receptor signaling. Cell 122: 835-847, 2005.

27. Tajeddine N, Gala JL, Louis M, Van Schoor M, Tombal B and Gailly P: Tumor-associated antigen preferentially expressed antigen of melanoma (PRAME) induces caspase-independent cell death in vitro and reduces tumorigenicity in vivo. Cancer Res 65: 7348-7355, 2005.

28. Tanaka N, Wang YH, Shiseki M, Takanashi M and Motoji T: Inhibition of PRAME expression causes cell cycle arrest and apoptosis in leukemic cells. Leuk Res 35: 1219-1225, 2011.

29. Seven D, Yavuz E, Kilic E, Baltaci E, Karaman E, Ulutin T and Buyru N: DLEC1 is not silenced solely by promoter methylation in head and neck squamous cell carcinoma. Gene 563: 83-86, 2015.

30. Murr R: Interplay between different epigenetic modifications and mechanisms. Adv Genet 70: 101-141, 2010.

31. Pampalakis G and Sotiropoulou G: Multiple mechanisms underlie the aberrant expression of the human kallikrein 6 gene in breast cancer. Biol Chem 387: 773-782, 2006.

32. Li Y, Li S, Chen J, Shao T, Jiang C, Wang Y, Chen H, Xu J and Li X: Comparative epigenetic analyses reveal distinct patterns of oncogenic pathways activation in breast cancer subtypes. Hum Mol Genet 23: 5378-5393, 2014. 